\title{
A!
}

This is an electronic reprint of the original article.

This reprint may differ from the original in pagination and typographic detail.

Vierros, Sampsa; Sammalkorpi, Maria

\section{Hybrid Atomistic and Coarse-Grained Model for Surfactants in Apolar Solvents}

\section{Published in:}

ACS Omega

DOI:

10.1021/acsomega.9b01959

Published: 01/01/2019

Document Version

Publisher's PDF, also known as Version of record

Published under the following license:

CC BY

Please cite the original version:

Vierros, S., \& Sammalkorpi, M. (2019). Hybrid Atomistic and Coarse-Grained Model for Surfactants in Apolar Solvents. ACS Omega, 4(13). https://doi.org/10.1021/acsomega.9b01959

This material is protected by copyright and other intellectual property rights, and duplication or sale of all or part of any of the repository collections is not permitted, except that material may be duplicated by you for your research use or educational purposes in electronic or print form. You must obtain permission for any other use. Electronic or print copies may not be offered, whether for sale or otherwise to anyone who is not an authorised user. 


\title{
Hybrid Atomistic and Coarse-Grained Model for Surfactants in Apolar Solvents
}

\author{
Sampsa Vierros ${ }^{\dagger}$ and Maria Sammalkorpi* ${ }^{\dagger} \dagger, \ddagger$ (0) \\ ${ }^{\dagger}$ Department of Chemistry and Materials Science and ${ }^{\ddagger}$ Department of Biomaterials and Bioproducts, Aalto University, P. O. Box \\ 16100, 00076 Aalto, Finland
}

\section{Supporting Information}

\begin{abstract}
Here, we develop and verify the performance of a hybrid molecular modeling approach that combines coarse-grained apolar solvent and atomistic solute or polar solvent description, for example, for description of reverse micellar systems. The coarse-grained solvent model is directly applicable to organic solvents encompassing alkane, alkene, and fatty acid ester functional groups and connects directly to both standard united-atom GROMOS 53A6 and all-atom CHARMM27 force fields, as well as the atomistic detail water models compatible with these force fields. The different levels of description are coupled via explicit, unscaled electrostatics, and scaled mixing rules for dispersive interactions. The hybrid model

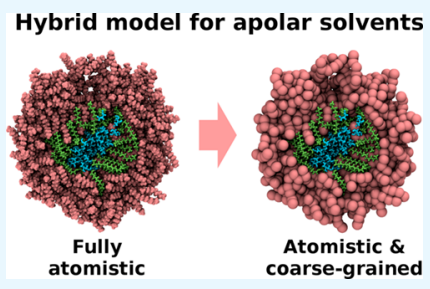
is in near-quantitative agreement with fully atomistic simulations when combined with the CHARMM27 model but underestimates modestly surfactant aggregation when using GROMOS 53A6 united-atom description. The use of truncated electrostatics affords up to a 9-fold increase in computational speed without significant loss of accuracy. However, long-range electrostatic calculations and load imbalance at high core counts can significantly degrade the performance. We demonstrate the usability of the hybrid model by assessing the reverse micelle formation of a homologous series of nonionic glycerolipids via large-scale self-assembly simulations. The presented model is demonstrated here for accurate description of surfactant systems in apolar solvents, with and without also polar solvent (water) in the system. The formulation can be expected to describe well also other solute species or interfaces with an apolar solvent in an apolar environment.
\end{abstract}

\section{INTRODUCTION}

Surfactants in apolar solvents form reverse micellar and emulsion systems that are both technologically and fundamentally significant. They are commonly used as highly tunable and dynamic platforms for drug delivery, ${ }^{1}$ protein separation, ${ }^{2}$ and templates for inorganic nanoparticle synthesis. $^{3}$ Additionally, surfactant-apolar solvent systems are present and have an important role in cosmetics, paints, biobased oils, and alimentation. ${ }^{4}$ At the same time, the often significant sensitivity to microscopic, localized detail level interactions, for example, solvent quality and hydrogen bonding, ${ }^{5-7}$ makes them interesting from a theoretical standpoint.

The modeling of apolar surfactant systems using current atomistic molecular models is limited in the attainable size and timescales by the computationally taxing nature of atomistic force fields, as well as by the special characteristics of apolar systems, for example, long relaxation times resulting from high solvent viscosity. Coarse-grained modeling approaches, which group neighboring atoms into single interaction sites, provide access to longer characteristic relaxation times and larger system sizes. Coarse-grained approaches have enabled, for example, large-scale simulations of lipid membranes, ${ }^{8,9}$ protein systems, ${ }^{10}$ and predicting structures of polymer systems. ${ }^{11,12}$ However, localized specific interactions, charge correlations, and sensitivity to, for example, hydrogen bonding, which are all often observed in apolar surfactant systems, are challenging for coarse-grained interaction models to capture. This results from the concept relying on employing effective, delocalized interactions to capture molecular system performance.

A computationally efficient, accurate simulation model for apolar systems is needed because the soft, noncrystalline nature of the aggregates formed by surfactants in apolar solvents, in particular reverse micelles, makes them difficult to study experimentally. While the structure of the aggregates can be studied using, for example, scattering techniques ${ }^{13,14}$ and various probe-based and spectroscopic methods exist to characterize the dynamics and structuring inside the polar cores, ${ }^{15,16}$ the resulting data often paints out a smeared picture of the underlying molecular-level structure and dynamics.

Here, we develop a hybrid model that is particularly aimed at bridging the gap between atomistic and coarse-grained modeling to provide accurate, enhanced computational efficiency description of surfactants in apolar solvents. The model uses supra-atomic coarse-grained detail for the apolar solvent environment yet retains atomistic detail for those molecular species that bear the features leading to the microscopic detail sensitivity in the structure and dynamics of these systems. The supra-atomic coarse-graining approach is similar to most coarse-grained models with the exception that water is usually coarse-grained supramolecularly.

Received: June 29, 2019

Accepted: September 3, 2019

Published: September 13, 2019 
The key challenge in hybrid molecular modeling is how to couple the atomistic and coarse-grained species together. The approaches taken in the literature vary and mostly concentrate on aqueous systems. For instance, for their PACE protein model, Han and co-workers ${ }^{17-20}$ opted to explicitly parametrize the all-atom/coarse-grained (AA/CG) cross-interactions against small-molecule hydration data. The approach enabled affordable aqueous folding simulations of several small proteins. Rzepiela et al. ${ }^{21}$ and later Wassenaar et al. ${ }^{22}$ coupled the coarse-grained MARTINI ${ }^{23}$ model with the atomistic GROMOS force field ${ }^{24}$ by embedding atomistic solutes inside a virtual coarse-grained representation of the said solute, while another approach was utilized by Riniker et al. ${ }^{25,26}$ and the developers of the ELBA model, ${ }^{27,28}$ who derived parameters for the AA/CG interactions using standard mixing rules. However, the authors found that scaling the resulting parameters was necessary for accuracy. Notably, Orsi et al. have presented a hybrid approach for coarse-grained water solvating atomistic detail solutes where the accuracy persists without scaling. ${ }^{29,30}$ Finally, perhaps the most complicated and ambitious approach taken is the so called adaptive resolution methods. The above examples all utilize a static, moleculebased division between coarse-grained and fine-grained components, but in the so called AdResS approach, solvent molecules are allowed to change dynamically between different representations based on, for example, whether or not the molecule is considered bulk-like or interfacial. With this approach, any challenges in coupling a coarse-grained model to fine-grained features of the system are largely avoided (see, for example, refs 31-33).

Also, for surfactants in apolar solvents, namely, reverse micelles, hybrid modeling approaches have been employed a handful of times. Brodskaya and Mudzhikova ${ }^{34-36}$ coarsegrained both the surfactant and the solvent but retained atomistic representation of water and counterions to model the aqueous core of AOT reverse micelles. The coupling between atomistic and coarse-grained parts of the system was achieved through combination rules. This approach was also taken by Brown and Clarke, ${ }^{37}$ although at a considerably coarser scale and a separately parametrized coupling scheme. Nevidimov and Razumov ${ }^{38,39}$ chose to apply coarse-graining only to the apolar solvent, while the surfactant, water, and ions were represented in full atomistic detail. This enabled them to undertake large-scale self-assembly simulations of AOT reverse micelles. ${ }^{39}$ Furthermore, even though the approach can still be considered fully atomistic modeling, Braun et al. ${ }^{40}$ and Schmollngruber et al. $^{41}$ have successfully combined unitedatom and all-atom descriptions to model AOT reverse micellar systems.

The model we develop here is a general apolar solvent coarse-grained model that encompasses hydrocarbon and ester group containing species as a solvent environment. In particular, the coarse-grained model focuses on description of biologically relevant compounds, such as triglycerides and fatty acid esters, because of the increasing interest in using biobased compounds in formulations. The model can be combined with existing all-atom detail models for surfactants or other solutes including atomistic detail water and ions, which gives it a very wide usability range. Furthermore, the proposed coarse-grained model reproduces to a large part the energetics and characteristics of fully atomistic models for the solvent species by explicitly accounting for hydrogen bonding via the use of dipolar sites. We test the coupling of the coarse-grained model to atomistic models with two commonly used force fields of different levels of description, namely, united-atom GROMOS $56 \mathrm{~A}^{24}$ and explicit-hydrogen CHARMM27, ${ }^{42}$ and benchmark the resulting hybrid model for computational performance. Finally, we demonstrate the use and usability of the new hybrid description via examining the dependence of the aggregation mechanism on the surfactant structure for a homologous, systematic series of nonionic glycerolipids in an apolar triglyceride solvent. The current work exceeds the prior efforts in (1) generality, that is, it covers a wider range of apolar solvents than only model hydrocarbon solvents and covers also complex biological apolar solvents, (2) the usability range, as it is fully compatible with existing major atomistic detail force fields including water models, and (3) also the extent of model verification, which covers both molecular characteristics and self-assembly response of a set of surfactants.

\section{METHODS}

Parametrization. The supra-atomic coarse-grained (CG) solvent model follows roughly a 3-to-1 mapping: on average, 3 connected heavy atoms and the associated hydrogens are represented by a single CG interaction site (bead). To account for the strong dipoles of ester groups, these additionally carry an off-center charged particle. To reduce the overall complexity of the model, a minimal number of beads is used. Except for the number of mapped hydrogens, each CG bead corresponds to a well-defined target structure at the atomistic detail level. This means that, for example, three methylene groups, two methylene groups terminated by a methyl group, and the propyl backbone of triglycerides are all represented by the same bead type. The adopted approach is similar to, for example, the MARTINI model ${ }^{23}$ but different from the $\mathrm{SDK}^{43}$ or the GROMOS alkane $\mathrm{CG}^{44}$ models, which differentiate between chain middle and terminal alkane beads. The here adopted approach attempts to retain the simplicity of the MARTINI model while aspiring for the chemical accuracy of the more complicated models. The mapping choice is illustrated in Figure 1.

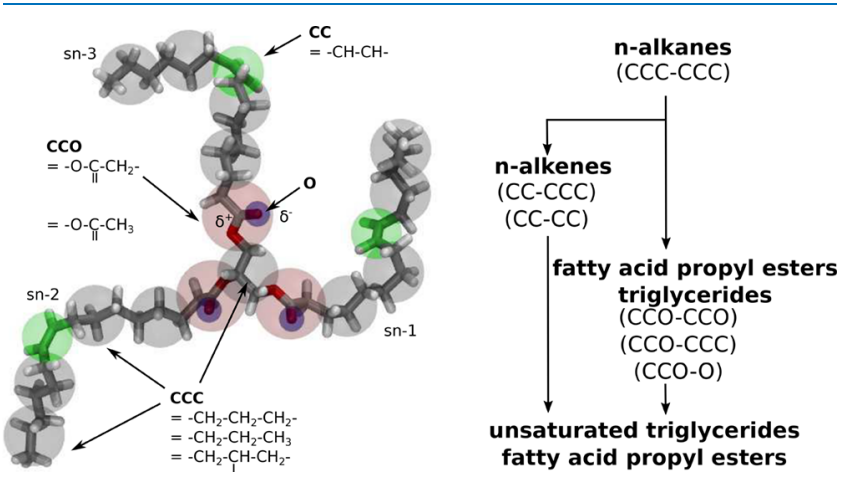

Figure 1. On the left, the beads of the coarse-grained model and their mapping to atomistic structure of tripalmitolein as an example. On the right, the hierarchy of model compounds (bold) and interactions (in parentheses) on which the parametrization is based.

For compatibility with common empirical biomolecular force fields and simulation packages, the potential energy function of the coarse-grained model consists of harmonic bond potentials combined with the Lennard-Jones and Coulomb potentials for the nonbonded interactions. Bonded 
interactions are modeled using harmonic bond stretching and angle bending terms

$$
U_{\text {bonded }}=\sum^{\text {bonds }} \frac{k_{\mathrm{b}}}{2}\left(b-b_{0}\right)^{2}+\sum^{\text {angles }} \frac{k_{\theta}}{2}\left(\theta-\theta_{0}\right)^{2}
$$

where $k_{\mathrm{b}}$ and $k_{\theta}$ are force constants and $b_{0}$ and $\theta_{0}$ are the equilibrium values for the bond stretching and angle bending terms, respectively. Bonded parameters are obtained via matching the resulting equilibrium distance and angle distributions with reference distributions obtained via center of mass based coarse-graining of fine-grained, atomistic detail trajectories calculated for target molecules. The CG model does not contain a specific term for torsions as a good match with atomistic distributions for the parametrized species is obtained even with only bond streching and bending terms (see the Supporting Information for data). We point out that for molecular species containing strong intramolecular interactions, torsions may be necessary in the CG representation.

Nonbonded interactions are given by

$$
\left.U_{\text {nonbonded }}=\sum 4 \epsilon_{i j}\left(\frac{\sigma_{i j}}{r_{i j}}\right)^{12}-\left(\frac{\sigma_{i j}}{r_{i j}}\right)^{6}\right\}+\sum^{\text {Coulomb }} \frac{1}{4 \pi \epsilon} \frac{q_{i} q_{j}}{r_{i j}}
$$

where $\epsilon_{i j}$ and $\sigma_{i j}$ are the potential well-depth and zero distance of the Lennard-Jones potential and $q$ is the charge of the particle. Nonbonded interactions between $1-2$ bonded pairs are excluded in the model. The Lennard-Jones parameters were fitted to reproduce the liquid density and heat of vaporization of the model compounds, while the Coulomb interactions of hydrogen bonding species were fitted to match the binding energy defined based on atomistic simulation PMFs. Liquid density was evaluated directly from a bulk NPT simulation, while the heat of vaporization $\Delta H_{\mathrm{m}}$ was calculated according to

$$
\Delta H_{\mathrm{m}}=\left(E_{\mathrm{pot}}(\mathrm{g})+R T\right)-E_{\mathrm{pot}}(\mathrm{l})
$$

where $E_{\text {pot }}(\mathrm{g})$ and $E_{\text {pot }}(1)$ are the potential energies per molecule in the gas phase and liquid phase, respectively, and $R T$ corresponds to thermal energy. The gas-phase potential energy $E_{\text {pot }}(g)$ was evaluated from a separate gas-phase simulation.

The parametrization of the nonbonded LJ interactions was conducted manually against a homologous series of model compounds. The parameter adjustment was conducted in a hierarchical manner; that is the simpler compounds such as alkanes were parameterized first after which the obtained alkane parameters were used without further adjustment in the more complex molecules (see Figure 1). All cross-interactions were not explicitly parametrized; for example, the CCO-CC interaction had relatively little impact on the properties of unsaturated esters and glycerides, and because of this, its magnitude was determined via Lorentz-Berthelot combination rules. Although the parametrization here was done manually, the findings point that this tuning process could be automated, as, for example, Reith et al. have demonstrated. $^{45}$ However, some level of manual assessment of the system is advisable to prevent overfitting the model. The parametrization hierarchy and an explicit listing of the beads and corresponding all-atom chemical structures are presented in Figure 1.
Prior to general nonbonded parametrization, the structure and electrostatic properties of the coarse-grained ester group were devised. To ease the task, parametrization effort focused on the carbonyl dipole: the off-center charged particle, named $\mathrm{O}$, was set to correspond to the carbonyl oxygen. The position of the particle was determined based on the distance from the carbonyl oxygen to the group center of mass in atomistic simulations $(0.132 \mathrm{~nm})$. The mass of the particle was set as $16 \mathrm{u}$, the mass of oxygen. The rest of the ester group mass was partitioned to the parent bead. The geometry of the ester offcenter charge at $\mathrm{O}$ is kept in place by a distance constraint to the ester bead $\mathrm{CCO}-\mathrm{O}$ and two angle bending terms to the adjacent beads CCC-CCO-O and O-CCO-CCC. The strength of the carbonyl dipole and the Lennard-Jones parameters of the particle $\mathrm{O}$ were initially borrowed from the GROMOS 53A6 model. Subsequently, these were periodically adjusted during the nonbonded parametrization to reproduce the $\Delta G$ and equilibrium distance of the ester-alcohol free energy profile at hybrid modeling resolution. As the hybrid scheme uses direct electrostatic coupling $\left(\epsilon_{\mathrm{r}}^{\mathrm{AA} / \mathrm{CG}}=1\right)$ between atomistic and coarse-grained species, the parametrization of the ester bead effectively fixes the CG/AA electrostatic coupling.

The CG/AA Lennard-Jones parameters were calculated using the Lorentz-Berthelot mixing rules with additional scaling applied to the well-depth $\epsilon$. The scaling factor was applied to all CG/AA interaction uniformly, but the value of it was determined separately for the coupling of C27 and 53A6 models. The optimal value of the scaling factor was obtained by matching the free energy profile of pulling two atomistic dodecane molecules apart in coarse-grained bulk dodecane to a fully atomistic reference energy profile.

Fine-Grained Simulations. United-atom GROMOS $53 \mathrm{~A}^{24}$ and all-atom CHARMM C2 $7^{42}$ force fields were used for the fine-grained part of the hybrid model. These versions of the respective models (as opposed to, for example, C36 or 53A6) were chosen based on prior experience with CHARMM force fields ${ }^{46}$ and preliminary benchmarking of binding energies against the CHARMM Drude polarizable force field. ${ }^{47}$ van der Waals interactions were smoothly switched to zero between 1.0 and $1.2 \mathrm{~nm}$ (C27) or cut at $1.4 \mathrm{~nm}$ (53A6). Tail correction was applied for C27. Electrostatics were handled either by the smooth particle mesh Ewald (PME) method ${ }^{48}$ (fourth-order interpolation, 0.1 $\mathrm{nm}$ grid spacing $)$ or reaction-field electrostatics $\left(\epsilon_{\mathrm{r}}=1, \epsilon_{\mathrm{RF}}=\right.$ $\infty)$. Infinite screening is used so that the atomistic and hybrid simulations can employ the same parameters; notably, the chosen cutoff scheme reproduces nearly exactly atomistic PMFs calculated using PME electrostatics for polar compounds. All bulk simulations employed PME, while the selfassembly simulations made use of reaction-field electrostatics and PME in separate simulations. LINCS $^{49}$ was used for all non-water distance constraints for all bonds (53A6) or bonds containing hydrogen (C27). SETTLE ${ }^{50}$ was used to keep the water molecules rigid. These constraint approaches allow using an integration timestep of $2 \mathrm{fs}$. The stochastic velocity rescaling thermostat of Bussi et al. ${ }^{51}\left(\tau_{T}=0.5 \mathrm{ps}\right.$ and $\left.T_{\text {ref }}=298.15 \mathrm{~K}\right)$ and Parinello-Rahman barostat ${ }^{52}\left(\tau_{P}=2\right.$ ps and $P_{\text {ref }}=1$ bar $)$ were used to implement constant pressure and temperature conditions. The above parameters were used in the umbrella simulations as well. In gas-phase simulations, all interactions were included, pressure coupling was turned off, and the stochastic dynamics thermostat was used. See Section S1 of the 
Supporting Information for detailed information on the finegrained system setup and compositions.

Coarse-Grained and Hybrid Simulations. Parametrization of the coarse-grained model employed 53A6 fine-grain simulation parameters with the following exceptions: an analytical tail correction was applied to the Lennard-Jones potential to ensure a cutoff independent density and heat of vaporization, and with the exception of bonds connecting offcenter charges to ester groups, all coarse-grained bonds were flexible. In the hybrid simulations, simulation parameters of the associated fine-grained model were used with the above listed exceptions.

Umbrella Sampling Simulations. Umbrella sampling was used to determine the potential of mean force (PMF) to estimate the free energy profiles between various species. Starting configurations for the umbrella sampling windows were generated by pulling two binding species apart at a rate of $0.002 \mathrm{~nm} / \mathrm{ps}$. Approximately 5-10 windows were created at $\sim 0.2 \mathrm{~nm}$ intervals. Each window was simulated for $40 \mathrm{~ns}$ (500 ns for the dodecane in dodecane PMF), and the PMF was extracted using the weighted histogram analysis method. ${ }^{53,54} \mathrm{~A}$ force constant of $500 \mathrm{~kJ} \mathrm{~mol}^{-1} \mathrm{~nm}^{-2}$ was used for the harmonic umbrella potential. The PMF estimates presented in this work include the $2 R T \ln (z)$ Jacobian entropy correction. ${ }^{55}$

Benchmarks. The step-to-step performances of atomistic and hybrid resolution simulations were measured using monopalmitolein/dodecane systems. Final configurations of $200 \mathrm{~ns}$ atomistic self-assembly simulations were used as initial configurations and subsequently converted to hybrid resolution to eliminate any bias from differing starting configurations. Benchmarks were run on full nodes of the CSC Sisu supercomputer with each node consisting of 24 Intel Haswell cores (E5-2690v3, $2.6 \mathrm{GHz})$. Each simulation was run for at least 10000 steps, and cycle counters were reset halfway.

\section{RESULTS AND DISCUSSION}

Coarse-Grained Solvent Model. Table 1 lists the coarsegrained model parameters, while Figure 2 compares the density and heat of vaporization of the employed parametrization target molecules to experimental and simulated reference data (see Table S1 in the Supporting Information for reference data and Table S2 for numerical data on properties of coarsegrained model compounds). The data shows that the presented coarse-grained model parameters lead to target properties of pure hydrocarbons being well reproduced. However, for esters, a set of parameters, which would perfectly capture the entire target data set, could not be found, and the presented parameters are a compromise. Specifically, perfectly matching the properties of monoesters resulted in overestimation of triglyceride $H_{\mathrm{m}}$. Hence, the cross-interaction between hydrocarbon and ester beads was scaled down, which leads to the coarse-grained model systematically underestimating monoester densities but resulting in an improved fit for the triglycerides. This challenge in ester parametrization could result from the simplicity of the model, which may prevent reproducing both triglyceride and monoester properties using the same parameters. This could very likely be resolved with separate parametrization; however, here, we have settled on the aforementioned compromise in the interest of simplicity and since experimental triglyceride $H_{\mathrm{m}}$ values suffer from discrepancies. These discrepancies could not be resolved with atomistic reference data either due to differing predictions made by $\mathrm{C} 27$ and 53A6. More detailed data about the
Table 1. Summary of the Coarse-Grained Parameters ${ }^{a}$

\begin{tabular}{|c|c|c|c|c|}
\hline \multicolumn{5}{|c|}{ bond type } \\
\hline$i$ & $j$ & & $b_{0}(\mathrm{~nm})$ & $k_{\mathrm{b}}\left(\mathrm{kJ} \mathrm{mol} \mathrm{mm}^{-1} \mathrm{~nm}^{-2}\right)$ \\
\hline $\mathrm{CCC}$ & $\mathrm{CCO}$ & & 0.344 & 6000 \\
\hline $\mathrm{CCO}$ & $\mathrm{CCC}$ & & 0.344 & 5000 \\
\hline $\mathrm{CCC}$ & $\mathrm{CCC}$ & & 0.365 & 5000 \\
\hline CCC & $\mathrm{CC}$ & & 0.300 & 8000 \\
\hline $\mathrm{CCC}$ & $\mathrm{CCO}$ & $(s n-2)$ & 0.283 & 35000 \\
\hline $\mathrm{CCO}$ & $\mathrm{O}$ & & 0.132 & constraint \\
\hline \multicolumn{5}{|c|}{ angle type } \\
\hline$i$ & $j$ & $k$ & $\theta_{0}\left({ }^{\circ}\right)$ & $k_{\theta}\left(\mathrm{kJ} \cdot \mathrm{mol}^{-1} \cdot \operatorname{rad}^{-2}\right)$ \\
\hline $\mathrm{CCC}$ & $\mathrm{CCO}$ & $\mathrm{CCC}$ & 180 & 4.0 \\
\hline $\mathrm{CCO}$ & CCC & CCC & 180 & 8.0 \\
\hline $\mathrm{CCC}$ & $\mathrm{CCC}$ & CCC & 180 & 8.0 \\
\hline $\mathrm{CCC}$ & $\mathrm{CCC}$ & $\mathrm{CC}$ & 180 & 8.0 \\
\hline $\mathrm{CCC}$ & $\mathrm{CC}$ & $\mathrm{CCC}$ & 112 & 60.0 \\
\hline $\mathrm{CCC}$ & $\mathrm{CCO}$ & $\mathrm{O}$ & 90 & 75.0 \\
\hline $\mathrm{O}$ & $\mathrm{CCO}$ & $\mathrm{CCC}$ & 90 & 4.0 \\
\hline $\mathrm{CCO}$ & $\mathrm{CCC}$ & $\mathrm{CCO}$ & 120 & 8.0 \\
\hline \multicolumn{5}{|c|}{ vdW type } \\
\hline$i$ & & $j$ & $\sigma_{i j}(\mathrm{~nm})$ & $\varepsilon_{i j}\left(\mathrm{~kJ} \cdot \mathrm{mol}^{-1}\right)$ \\
\hline $\mathrm{CCO}$ & & $\mathrm{CCO}$ & 0.438 & 1.40 \\
\hline $\mathrm{CC}$ & & $\mathrm{CC}$ & 0.414 & 1.60 \\
\hline $\mathrm{CCC}$ & & $\mathrm{CCC}$ & 0.464 & 2.30 \\
\hline $\mathrm{O}$ & & $\mathrm{O}$ & 0.289 & 0.50 \\
\hline $\mathrm{CC}$ & & $\mathrm{CCC}$ & 0.440 & 1.75 \\
\hline $\mathrm{CCO}$ & & $\mathrm{CCC}$ & 0.446 & 1.41 \\
\hline $\mathrm{CCO}$ & & $\mathrm{O}$ & 0.415 & 1.02 \\
\hline
\end{tabular}
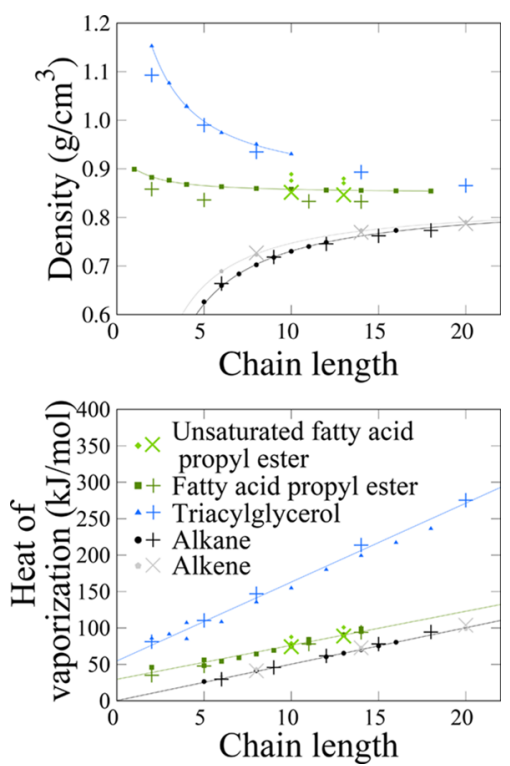

Figure 2. Comparison of liquid density and enthalpy of vaporization $H_{\mathrm{m}}$ calculated using the coarse-grained model and parametrization reference data. The symbols correspond to the coarse-grained model, and the solid lines correspond to experimental target data or, when unavailable, data computed in this work using the GROMOS 53A6 atomistic model. Target data points have been derived based on refs 56-62; see Table S1 in the Supporting Information for details.

parametrization is provided in the Supporting Information. In particular, Figure S1 compares bonded bead distances and 

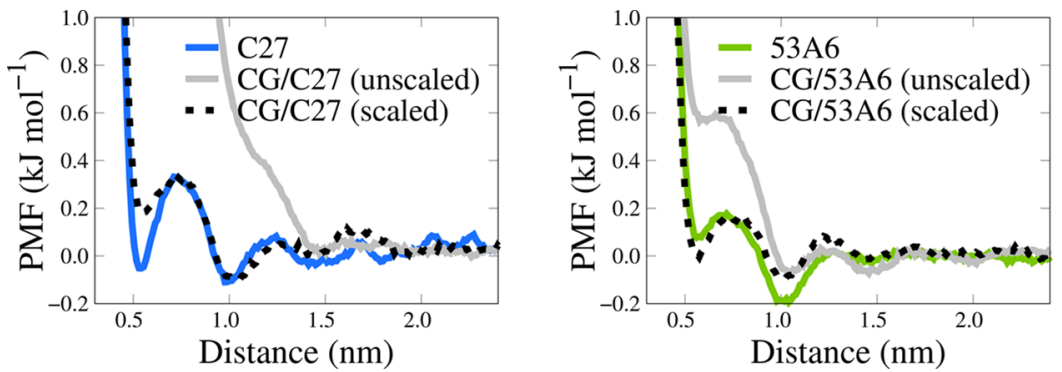

Figure 3. Potential of mean force (PMF) calculated for the center of mass separation distance of two dodecane molecules described in atomistic detail and dissolved in atomistic and coarse-grained dodecane. Results for both unscaled and scaled mixing rules are shown. On the left is data for CHARMM C27, and on the right is data for GROMOS 53A6 models.

angles in atomistic data with those resulting from the coarsegrained model parameters presented in Table 1.

Dispersive CG/AA Interactions. Next, the compatibility of the coarse-grained model with all-atom species is examined. In this, we take surfactants in apolar hydrocarbon solvents, that is, reverse micellar systems, as the target parametrization system. A common feature for many surfactant-apolar solvent systems, that is, reverse micellar systems, is that both the surfactants and the solvents are quite often based on aliphatic carbon chains. Specifically, most surfactants feature one or two hydrocarbon tails, while many common apolar solvents range from plain alkanes to hydrocarbons decorated with polar groups, for example, alcohols and esters. Even for complex solvents such as triglycerides, the main interaction between surfactant-like solute species and the solvent usually is via the hydrocarbon parts of the surfactant and the solvent. This leads to the paramount importance of accurate reproduction of the mixing between all-atom hydrocarbons (surfactant) and coarse-grained hydrocarbons (solvent).

To calibrate the coupling between atomistic and coarsegrained species, we calculated the free energy difference associated with separating two atomistic dodecane molecules dissolved in atomistic and coarse-grained dodecane (see Figure 3). The data shows that utilizing standard Lorentz-Berthelot mixing rules leads to the atomistic dodecane molecules preferentially dissolving in the coarse-grained solvent. While in the case of the united-atom 53A6 model combined with the coarse-grained solvent, the free energy difference of the first minima and complete separation is only slightly overestimated in comparison to atomistic simulations by $0.4-0.5 \mathrm{~kJ} / \mathrm{mol}$, and the association energy is substantially overestimated with the all-atom C27 model $(4.7 \mathrm{~kJ} / \mathrm{mol})$. However, scaling the $\epsilon$ parameter with a model dependent factor $\beta$ appears to largely recover the atomistic free energy landscape in both cases. We conclude that an optimal performance is obtained if factors $\beta=$ 0.975 and 0.847 for 53A6 and $\mathrm{C} 27$ are used, respectively. To cut down the number of parametrized interactions, these scaling values based on pure alkanes are utilized for all atomistic/coarse-grained cross-interactions.

To test the quality and performance of the scaling scheme, the self-aggregation of monopalmitin in coarse-grained dodecane and in atomistic C27 or 53A6 dodecane was compared. Figure 4 shows the resulting aggregate sizes and their time evolution. The simulations were carried out at $70{ }^{\circ} \mathrm{C}$ due to the observed high aggregation propensity of 53A6 monopalmitin. The figure shows reverse micelles in the system at each time by their aggregation numbers. The hybrid simulations produce reverse micelles with aggregation numbers consistent with the atomistic simulations, as shown by the
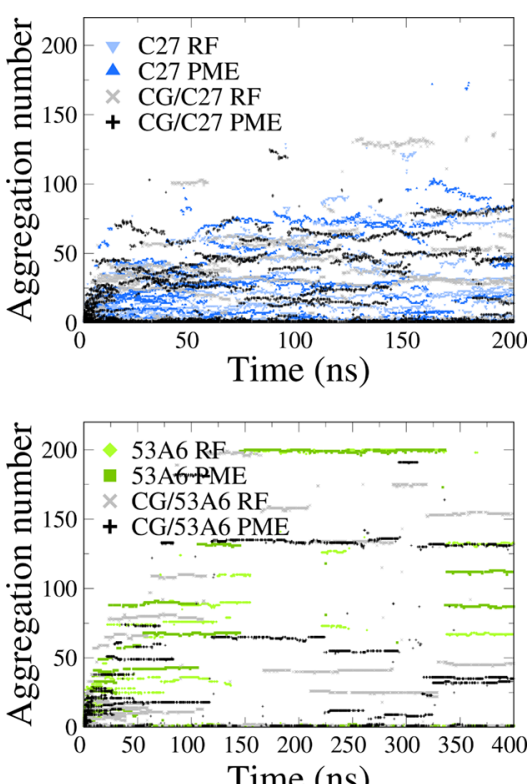

Time (ns)

Figure 4. Time evolution of aggregate size distributions in $12 \mathrm{wt} \%$ monopalmitin in dodecane solvent system using particle mesh Ewald (PME) and reaction-field electrostatics (RF). At the top is data for CHARMM C27, and at the bottom is data for GROMOS 53A6 atomistic models.

overlap of the distributions. The reverse micelles are predicted to be prolate (C27) or worm-like (53A6). Experimentally, Shrestha et al. ${ }^{5}$ have observed elongated monoglyceride reverse micelles suggesting that the simulation-predicted microstructure is qualitatively correct. The higher aggregation propensity predicted by the 53A6 model appears to result from the larger dipole moments in that model in comparison to $\mathrm{C} 27$ (see Figure 5 for energetics). Indeed, the partial charges of GROMOS 53A6 were parametrized to capture aqueous solvation energetics. ${ }^{24}$ The finding demonstrates that the implicit polarization of the underlying atomistic model is an important factor in reverse micellar simulations, at least in conditions corresponding to low hydration of the surfactant head groups.

The relatively good match between the atomistic and hybrid simulations is undoubtedly partially fortuitous, considering the higher temperature and small differences in the free energy profiles of Figure 3. To chart the hybrid model sensitivity to the scaling factor $\beta$, the size distribution dependency on $\beta$ was investigated for the C27 force field (see Figure S2 in the Supporting Information). The data shows that systematically higher aggregation numbers are favored when $\beta$ is smaller. A 

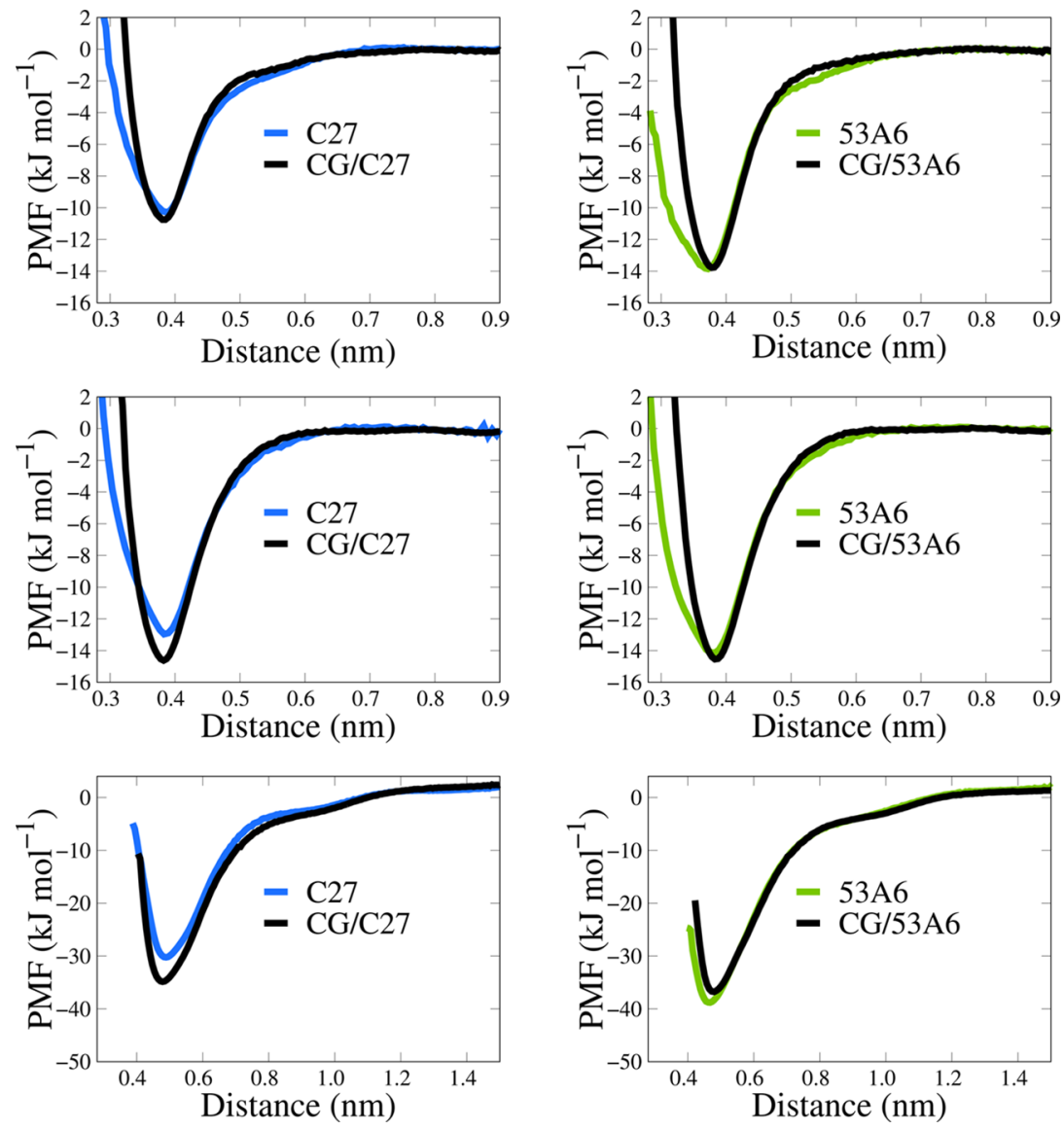

Figure 5. Comparison of potentials of mean force (PMFs) calculated using the atomistic CHARMM C27 or GROMOS 53A6 model and the hybrid coarse-grained CG/atomistic model. The presented PMFs are calculated between the distance of propyl myristate ester group and hexanol hydroxyl group (top row), water molecule (middle row), or tetramethylammonium ion in dodecane (bottom row). Short distance deviation can be explained by the CG bead size.

significant change occurs with only a $2 \%$ change in the coupling parameter. This suggests that the shape of the reverse micellar aggregate is very sensitive toward the tail-solvent mixing energy, both in all-atom and in the hybrid model; the finding is in qualitative agreement with the observations of Shrestha et al. ${ }^{5,63}$ and also our previous findings on phospholipid reverse micelles in cyclohexane. ${ }^{64,65}$ Given, however, that $\beta$ was fitted against the dodecane chain and still reproduces aggregate sizes of the longer-chain monopalmitin for both 53A6 and C27, we suggest that the presented $\beta$ parameters are balanced and that the $\beta$ parameter approach has a degree of transferability. Furthermore, application of the adaptive resolution technique in the form of a fine-grained solvent skin region could remove the sensitivity to the $\beta$ parameter. However, it is unclear how a large computational overhead maintaining such skin regions would introduce into the relatively packed reverse micellar system. Such an approach was not used here.

Electrostatic Coupling. Figure 5 shows the free energy profiles of propyl myristate (PM) binding with hexanol $(\mathrm{HxOH})$, water $\left(\mathrm{H}_{2} \mathrm{O}\right)$, and tetramethylammonium ion $\left(\mathrm{TMA}^{+}\right)$. The propyl myristate-hexanol binding energy was used in the parametrization of the coarse-grained ester group, while the latter two binding species were used to investigate the transferability of the parameters. In the potential of mean force profiles, the depth of the minimum corresponds to the binding free energy of the two species, that is, free energy difference corresponding to binding of the two compounds in the solvent. The data shows that, for charge neutral species, the hydrogen bonding energy in the hybrid simulations is reasonably well reproduced in comparison to the fully atomistic reference systems (within $1.6 \mathrm{~kJ} / \mathrm{mol}$ in all cases). However, the ion-dipole interaction energy of the PM/TMA ${ }^{+}$ complex is overestimated by $5 \mathrm{~kJ} / \mathrm{mol}$ for $\mathrm{C} 27$ and underestimated by $3 \mathrm{~kJ} / \mathrm{mol}$ for 53A6. The loci of the PMF minima are in similar acceptable agreement between the atomistic and hybrid simulations for both atomistic models. However, at close distances, the PMF rises too steeply in the hybrid simulations, which likely results from the inaccurate description of the shape anisotropy of the ester group at very short distances by the CG model. This could partly be remedied by manually adjusting the Lennard-Jones parameters between the coarse-grained ester group and the atomistic binding species. However, as the coarse-grained ester group lacks secondary dipoles, that is, additional charged particles, which would form weaker dipoles with the central charged bead, we did not pursue this here as it could lead to overfitting of the model.

To further validate the electrostatic coupling between atomistic and the coarse-grained descriptions in the hybrid model, a system of $21 \mathrm{wt} \%$ monopalmitin in bulk propyl decenoate at $25{ }^{\circ} \mathrm{C}$ was characterized in terms of aggregate size distributions. This solvent/surfactant pair has sufficiently fast dynamics even at room temperature for the system to reach a 

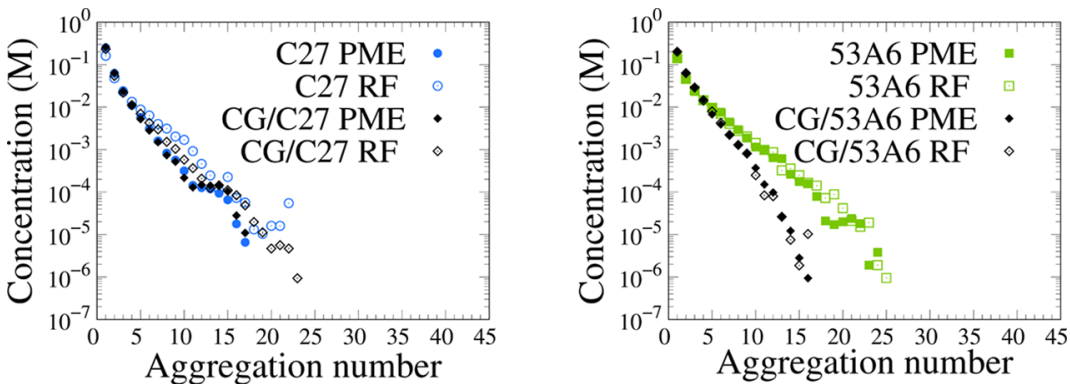

Figure 6. Aggregate size distributions in $21 \mathrm{wt} \%$ monopalmitolein/propyl decenoate system calculated using particle mesh Ewald (PME) and reaction-field $(\mathrm{RF})$ electrostatics.
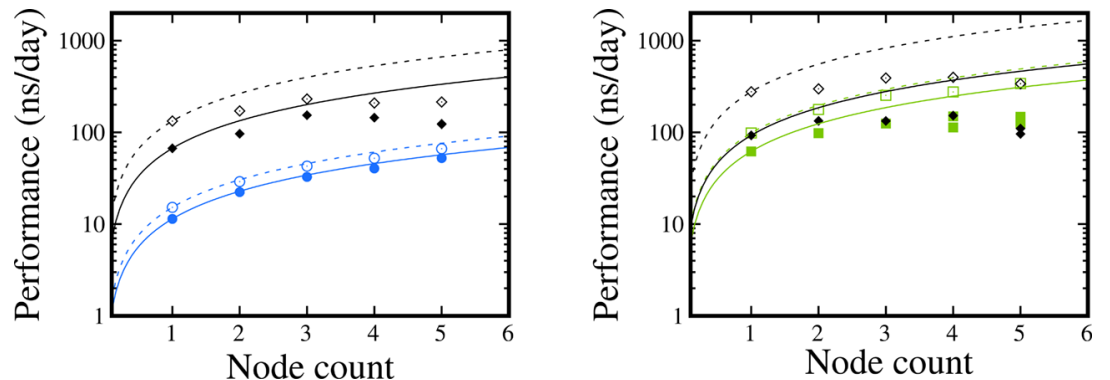

Figure 7. Benchmarking data for C27 (left) and 53A6 (right). In the figure, black corresponds to hybrid resolution, green to united-atom GROMOS 53A6 force field, and blue to all-atom C27 force field. Filled symbols and solid lines correspond to simulations with PME electrostatics, and empty symbols and dashed lines correspond to reaction-field electrostatics. Each node consists of 24 Intel Haswell cores (E5-2690v3, 2.6 $\mathrm{GHz})$.

converged size distribution within the simulation duration. The resulting size distributions are shown in Figure 6. The data shows that the aggregate size distribution is qualitatively reproduced for both $\mathrm{C} 27$ and 53A6 force fields. However, the free surfactant concentration is overestimated for both force fields. Furthermore, the hybrid 53A6 simulations underestimate the aggregation propensity producing too steeply decaying aggregate size distribution in comparison to fully finegrained reference data. This is rather curious since the free energy profiles presented in Figures 3 and 5 are generally better reproduced by the 53A6 model than by the C27 model. We speculate that the self-interaction energy of 53A6 propyl decenoate differs somewhat from that of the coarse-grained one leading to the observed deviations. This suggests that, for balanced interactions, hybrid models should use the target atomistic energetics rather than experimental data as the reference data.

Effect of Long-Range Electrostatics. It is well known that truncation of Coulomb interactions can lead to various simulation artifacts. $^{66-69}$ However, for computational efficiency, coarse-grained models commonly incorporate interactions that have electrostatic origin, that is, dipole-dipole and even charged interactions, into short-ranged effective potentials, which are then truncated at a suitable distance. The truncation is somewhat justified by the fast $r^{-6}$ decay of thermally averaged dipolar interactions and the strong charge screening in the aqueous environment. However, the low dielectric permittivity of apolar solvents and the coexistence of coarse-grained and atomistic species in the present hybrid simulations make it unclear how much long-range electrostatics contribute to the structural and dynamical characteristics of the simulated systems. The presented model will describe charged species well, as long as the charges are in a molecular environment enabling properly their screening. The selected reaction-field electrostatic scheme, with infinite screening at the cutoff, will cause deviations in the description of charged species interactions in the dominantly apolar surroundings where the Debye screening length is long.

The effect of neglecting long-range electrostatics was tested by recalculating the size distributions in Figures 4 and 6 using also truncated electrostatics in addition to the PME electrostatics. We chose reaction-field electrostatics (RF) with infinite screening beyond the cutoff as the truncation scheme due to its ability to reproduce hydrogen bonding PMFs calculated using PME almost exactly (present work, data not shown) and the energy conservation it affords over straight cutoff schemes in prior simulations. ${ }^{70}$ Figures 4 and 6 show that, in the test systems, PME and truncated electrostatics yield size distributions and aggregation kinetics in close agreement with one another-both for fine-grained and hybrid simulations. This suggests that truncated electrostatics can be used for neutral reverse micellar systems without compromising the accuracy of the system description. We note, however, that simulations of systems containing mobile ions such as Aerosol OT reverse micelles or charged lipids should probably employ PME electrostatics.

Performance. Next, the speedup over standard atomistic methodology, as well as the scaling characteristics of the hybrid resolution model, is assessed by comparing representative model systems at different levels of resolution. Additionally, the effect of truncating electrostatic interactions on computational performance is assessed.

To probe the maximum achievable speedup between atomistic and coarse-grained representations, a system of pure propyl myristate in its bulk state ( 3200 molecules) was simulated both in the coarse-grained and atomistic details. With PME electrostatics, the speedup varies in the range of $1.3-2.8$ and $4.2-13.3$ in comparison to united-atom 53A6 and 


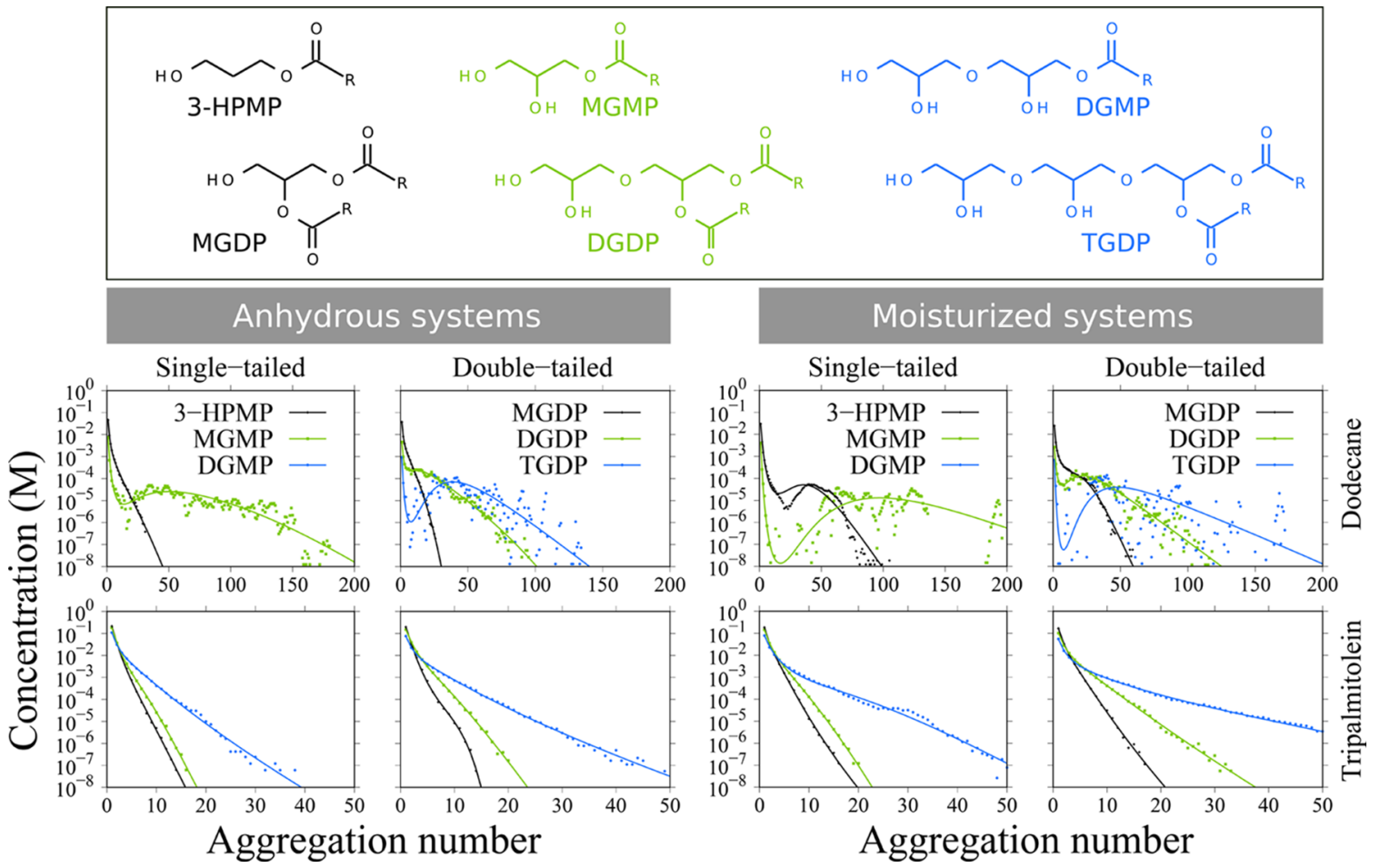

Figure 8. Examined single- and double-tailed glycerolipids (top) and their self-assembly size distributions in dodecane and tripalmitolein with (right panels) and without (left panels) residual moisture. Surfactant concentration varies in the ranges of 106-111 and 286-323 mM in dodecane and tripalmitolein, respectively. The corresponding water-to-surfactant ratios are $w_{0}=1$ and $w_{0}=2$. See Table S3 in the Supporting Information for detailed composition of each system. Solid lines correspond to smoothed size distributions; see Section S5 in the Supporting Information for description of the procedure.

all-atom C27 force fields, respectively. The use of truncated electrostatics increases the speedup factors to 4.1-4.8 and 21.3-31.6. The same electrostatics parameters are used when comparing models of different description levels; that is, also truncated electrostatic performance is compared between atomistic simulations using truncated electrostatics and hybrid CG-atomistic simulations using truncated electrostatics and correspondingly for PME performance comparison. In both cases, the performance advantage of the coarse-grained model decreases as more CPU cores are utilized. For truncated electrostatics, this effect appears to result from the natural scaling characteristics of the underlying simulation software; that is, the number of atoms per core slowly approaches the scaling limit. For PME, however, the coarse-grained simulations suffer from the mesh part of the PME algorithm becoming a bottleneck. This suggests that the best value for the CPU time for simulations using this hybrid model is obtained with low core counts and truncated electrostatics.

As a more realistic test case, the 12 wt \% monopalmitin/ dodecane self-assembly simulations presented in Figure 4 were also benchmarked (see Figure 7). With PME electrostatics, the relative performance varies between 0.7 and 1.5 and 2.3 and 5.9 for $53 \mathrm{~A} 6$ and $\mathrm{C} 27$, respectively. With truncation of electrostatics, these numbers increase to $1.0-2.8$ and 3.6-8.7, respectively. While the scaling characteristics mirror those obtained from the pure solvents, it is interesting to note that the hybrid simulations suffer at high node counts from severe load imbalance due to domain decomposition. This results from the heterogeneous particle density present in the system, that is, relatively sparse solvent, which atomistic reverse micelles of high particle density traverse randomly. Dealing with such a setup appears very difficult for the present load balancing algorithms.

The benchmarking data here corresponds to a formal stepto-step speedup factor, which neglects the artificial acceleration of diffusion processes resulting from coarse-graining. To investigate the significance of such acceleration in the hybrid simulations, we calculated the surfactant diffusion coefficients from the monopalmitolein/propyl decenoate simulations (the dodecane simulations yielded poor statistics due to the slowly diffusing aggregates). For the 53A6 model, surfactant diffusion was 1.7-1.8 times faster in the coarse-grained solvent, and for C27, the numbers were 3.7-5.2. For truncated electrostatics, the diffusion increase was less than for PME electrostatics. For C27, additionally, the diffusion of diglycerol monopalmitolein in tripalmitolein was assessed; surfactant diffusion was found to be 9 times faster than in atomistic detail simulations (see Figure S5 in the Supporting Information for size distribution comparison). This rather significant speedup suggest that, for triglycerides at least, switching to the coarse-grained solvent representation may be worthwhile even if there would not be other computational advantage.

Application: Self-Assembly of Glycerolipids in Apolar Solvents. Prior computational ${ }^{46}$ and experimental ${ }^{63,71-74}$ studies have suggested that the aggregation mechanism and aggregate morphology of nonionic surfactants in apolar media can be tuned by adjusting the polarities of the surfactant headgroup and solvent. Here, we apply the present hybrid model to a homologous series of glycerolipid surfactants containing 1-3 hydroxyl groups per headgroup and 1-2 hydrocarbon tails (see Figure 8 for the structures and abbreviations). The simulations are performed in both dodecane and tripalmitolein solvents in the presence and 
absence of moisture (water-to-surfactant molar ratio $w_{0}=1-$ 2). With this mapping, we aim to establish the conditions at which reverse micellization becomes favored over oligomerization.

Figure 8 presents the size distributions resulting from the simulations. Figures S3 and S4 in the Supporting Information show the corresponding time series. The prevalent association mechanism can be inferred from the shape of the size distribution: a straight line indicates classical indefinite, stepwise association, while curviness and the existence of a local minimum indicate cooperativity and a separate micellar population of preferred size (see ref 46 for more detailed discussion). The data shows that, in dodecane, the aggregation mode smoothly switches from stepwise association to cooperative reverse micellization as the headgroup polarity increases. The crossover from one mechanism to another appears for surfactants with two hydroxyl groups per headgroup with less polar surfactants following stepwise association and more polar surfactant cooperative association. For diglycerol monopalmitolein, the cooperativity of the aggregation was comparable to aqueous systems, and a reliable estimate for the size distribution could not be obtained. Contrary to this, in a tripalmitolein environment, all studied surfactants associate via the oligomerization mechanism. This is due to the hydrogen bonding ability of the solvent, which attenuates intersurfactant bonding. The data reveals both the significance of the hydrogen bonding capability of the surfactants in the formation of the reverse micelles but also the competing influence of the solvent hydrogen bonding ability. Similarly, solution additives with the hydrogen bonding ability can be expected to influence aggregation (see, for example, ref 75).

In dodecane, an increase in tail count decreases the size of the aggregates provided that the aggregation propensity is high enough for the formation of large reverse micelles. This behavior suggests that the increased steric repulsion due to two tails contributes significantly to aggregation only if a certain minimum level of crowdedness is met at the solvent/tail shell. Contrary to this hypothesis, in tripalmitolein, the double-tailed variants show a slightly higher overall aggregation propensity in comparison to single-tailed surfactants. This effect, however, appears to result from differences in headgroup size: at the relatively high surfactant concentration employed in the simulations, the elongated headgroups of diglycerol dipalmitolein (DGDP) and triglycerol dipalmitolein (TGDP) are more likely to reach an existing aggregate (and therefore be counted as a member) than their single-tailed variants (MGMP, DGMP). A preliminary set of simulations at approximately $120 \mathrm{mM}$ concentration showed the same trends, but much smaller differences between single- and double-tailed surfactants support this explanation.

Addition of water substantially increases the aggregation propensity in dodecane shifting all surfactants to favor cooperative reverse micellization over oligomerization (a separate population of reverse micelles emerges). The effect is, however, much more attenuated for double-tailed surfactants suggesting that the tail structure is an important control factor in reverse micellar systems. Visually, at these low hydration levels, water molecules are dispersed throughout the polar core region and only occasionally coalesce into a separate central water droplet. In tripalmitolein, addition of water increased the aggregation propensity slightly, but the aggregation mechanism remains essentially as oligomerization.
Shrestha et al. have conducted numerous X-ray scattering studies on the reverse micellization of nonionic glycerolipids. $^{63,71,76}$ While quantitative comparison cannot be made due to slight variations in tail structure, temperature, solvent chain length, and lack of moisture control, qualitatively, their observations are in agreement with the simulation results here. Namely, they report that the size of the reverse micelles increases with the headgroup size of the surfactant, ${ }^{71}$ while the increase in tail length ${ }^{76}$ or in the number of tails ${ }^{63}$ decreases the overall aggregate size. Addition of water was reported to increase the average aggregate size. ${ }^{71}$

\section{CONCLUSIONS}

In summary, we have developed a coarse-grained hydrocarbon solvent model that can be combined with existing atomistic detail models for both solutes and, for example, polar solvent species. We coupled the models with two atomistic force fields, namely, GROMOS 53A6 and CHARMM27, and verified the performance. The presented work encompasses both evaluation of the model construction and parametrization but also an assessment of the benefits and pitfalls of hybrid simulations in a reverse micellar setting. The findings suggest that, while good agreement between hybrid and atomistic simulations is achievable with relatively simple means, the direct coupling scheme employed in this paper also has some drawbacks. In particular, even though the hybrid scheme offers an increase in computational performance, scaling issues due to long-scale electrostatics and inhomogeneous particle density result in significant load imbalance at high core counts. This prevents the hybrid simulations from reaching substantially longer timescales than feasible with atomistic detail approaches. This problem is likely to affect all simulations using a similar coupling approach to this work.

We also report the aggregation responses of a homologous series of single- and double-tailed glycerolipids under various solvents and hydration conditions. The findings indicate that the headgroup polarity, particularly the hydrogen bonding donor capabilities of the headgroup, has a key role in determining the aggregation propensity of the surfactant. Further, water increases and surfactant hydrogen bonding with the solvent significantly decreases the cooperativity of the aggregation process. Finally, surfactant reverse micelles formed with single-tailed surfactants are significantly larger than those formed by surfactants with two tails. The presence of the second tail also promotes oligomerization as the aggregation mechanism. Altogether, the modeling work shows that, besides the usual oligomerization and cooperative association mechanisms, the reverse micellar systems also often bear characteristics of both of these; that is, the aggregation mechanism is mixed. This study is, to our knowledge, the first detailed computational investigation in which the mechanism of apolar aggregation is systematically evaluated. The work also presents for the first time with reasonable accuracy the shapes of the aggregate size distributions of reverse micelles of such glycerolipids or similar species. The results show that molecular dynamics simulations are able to provide detailed information on the aggregation response of surfactants also in these complex, dynamic apolar systems and to generate understanding of the nature of apolar solution surfactant aggregation. As the examined systems are of high chemical engineering importance, the findings could aid in tuning process solutions via engineering aggregate size distributions or enabling enhanced control of aggregation. 


\section{ASSOCIATED CONTENT}

\section{S Supporting Information}

The Supporting Information is available free of charge on the ACS Publications website at DOI: 10.1021/acsomega.9b01959.

Description of bulk simulations, list of self-assembly simulations and their compositions, sensitivity analysis of aggregate size on tail/solvent mixing energy, timedependent size distributions of glycerolipids, description of the aggregate size distribution smoothing scheme, and additional validation data (PDF)

\section{AUTHOR INFORMATION}

\section{Corresponding Author}

*E-mail: maria.sammalkorpi@aalto.fi.

\section{ORCID $\odot$}

Sampsa Vierros: 0000-0002-0656-4486

Maria Sammalkorpi: 0000-0002-9248-430X

\section{Notes}

The authors declare no competing financial interest.

\section{ACKNOWLEDGMENTS}

Funding from Aalto University School of Chemical Engineering Doctoral Programme is gratefully acknowledged (S.V.). The authors acknowledge the financial support of the Academy of Finland Grant No. 309324 (M.S.). We are grateful for the support by the FinnCERES Materials Bioeconomy Ecosystem. Computational resources by CSC IT Centre for Science, Finland, and RAMI - RawMatTERS Finland Infrastructure are also gratefully acknowledged.

\section{REFERENCES}

(1) Vintiloiu, A.; Leroux, J.-C. Organogels and their use in drug delivery - A review. J. Controlled Release 2008, 125, 179-192.

(2) Krishna, S. H.; Srinivas, N. D.; Raghavarao, K. S. M. S.; Karanth, N. G. Reverse Micellar Extraction for Downstream Processing of Proteins/Enzymes. Hist. Trends Bioprocess. Biotransform. 2002, 75, $119-183$.

(3) Chaurasiya, R. S.; Hebbar, H. U. Reverse Micelles for Nanoparticle Synthesis and Biomolecule Separation. In Nanoscience in Food and Agriculture 4 ; Ranjan, S., Dasgupta, N., Lichtfouse, E., Eds.; Springer International Publishing: Cham, Switzerland, 2017; pp 181-211.

(4) Svensson, M. Surfactants Based on Natural Fatty Acids. In Surfactants from Renewable Resources; Kjellin, M., Johansson, I., Eds.; Wiley-Blackwell: Chichester, West Sussex, UK, 2010; Chapter 1, pp $1-19$.

(5) Shrestha, L. K.; Glatter, O.; Aramaki, K. Structure of Nonionic Surfactant (Glycerol $\alpha$-Monomyristate) Micelles in Organic Solvents: A SAXS Study. J. Phys. Chem. B 2009, 113, 6290-6298.

(6) Shchipunov, Y. A.; Shumilina, E. V. Lecithin bridging by hydrogen bonds in the organogel. Mater. Sci. Eng. C 1995, 3, 43-50.

(7) Palazzo, G. Wormlike reverse micelles. Soft Matter 2013, 9, $10668-10677$.

(8) Wong-Ekkabut, J.; Baoukina, S.; Triampo, W.; Tang, I.-M.; Tieleman, D. P.; Monticelli, L. Computer simulation study of fullerene translocation through lipid membranes. Nat. Nanotechnol. 2008, 3, 363-368.

(9) Periole, X.; Knepp, A. M.; Sakmar, T. P.; Marrink, S. J.; Huber, T. Structural Determinants of the Supramolecular Organization of G Protein-Coupled Receptors in Bilayers. J. Am. Chem. Soc. 2012, 134, 10959-10965.
(10) Kmiecik, S.; Gront, D.; Kolinski, M.; Wieteska, L.; Dawid, A. E.; Kolinski, A. Coarse-Grained Protein Models and Their Applications. Chem. Rev. 2016, 116, 7898-7936.

(11) Kuo, A.-T.; Takeuchi, K.; Tanaka, A.; Urata, S.; Okazaki, S.; Shinoda, W. Exploring the effect of pendent side chain length on the structural and mechanical properties of hydrated perfluorosulfonic acid polymer membranes by molecular dynamics simulation. Polymer 2018, 146, 53-62.

(12) Srinivas, G.; Cheng, X.; Smith, J. C. A Solvent-Free Coarse Grain Model for Crystalline and Amorphous Cellulose Fibrils. J. Chem. Theory Comput. 2011, 7, 2539-2548.

(13) Schurtenberger, P.; Jerke, G.; Cavaco, C.; Pedersen, J. S. CrossSection Structure of Cylindrical and Polymer-like Micelles from Small-Angle Scattering Data. 2. Experimental Results. Langmuir 1996, 12, 2433-2440.

(14) Schurtenberger, P.; Cavaco, C. Polymer-like lecithin reverse micelles. 1. A light scattering study. Langmuir 1994, 10, 100-108.

(15) Willard, D. M.; Riter, R. E.; Levinger, N. E. Dynamics of Polar Solvation in Lecithin/Water/Cyclohexane Reverse Micelles. J. Am. Chem. Soc. 1998, 120, 4151-4160.

(16) Bakulin, A. A.; Cringus, D.; Pieniazek, P. A.; Skinner, J. L.; Jansen, T. L. C.; Pshenichnikov, M. S. Dynamics of Water Confined in Reversed Micelles: Multidimensional Vibrational Spectroscopy Study. J. Phys. Chem. B 2013, 117, 15545-15558.

(17) Han, W.; Wan, C.-K.; Jiang, F.; Wu, Y.-D. PACE Force Field for Protein Simulations. 1. Full Parameterization of Version 1 and Verification. J. Chem. Theory Comput. 2010, 6, 3373-3389.

(18) Han, W.; Wan, C.-K.; Wu, Y.-D. PACE Force Field for Protein Simulations. 2. Folding Simulations of Peptides. J. Chem. Theory Comput. 2010, 6, 3390-3402.

(19) Wan, C.-K.; Han, W.; Wu, Y.-D. Parameterization of PACE Force Field for Membrane Environment and Simulation of Helical Peptides and Helix-Helix Association. J. Chem. Theory Comput. 2012, 8, 300-313.

(20) Han, W.; Schulten, K. Further Optimization of a Hybrid United-Atom and Coarse-Grained Force Field for Folding Simulations: Improved Backbone Hydration and Interactions between Charged Side Chains. J. Chem. Theory Comput. 2012, 8, 4413-4424.

(21) Rzepiela, A. J.; Louhivuori, M.; Peter, C.; Marrink, S. J. Hybrid simulations: combining atomistic and coarse-grained force fields using virtual sites. Phys. Chem. Chem. Phys. 2011, 13, 10437-10448.

(22) Wassenaar, T. A.; Ingólfsson, H. I.; Prieß, M.; Marrink, S. J.; Schäfer, L. V. Mixing MARTINI: Electrostatic Coupling in Hybrid Atomistic-Coarse-Grained Biomolecular Simulations. J. Phys. Chem. B 2013, 117, 3516-3530.

(23) Marrink, S. J.; Risselada, H. J.; Yefimov, S.; Tieleman, D. P.; de Vries, A. H. The MARTINI Force Field: Coarse Grained Model for Biomolecular Simulations. J. Phys. Chem. B 2007, 111, 7812-7824.

(24) Oostenbrink, C.; Villa, A.; Mark, A. E.; van Gunsteren, W. F. A biomolecular force field based on the free enthalpy of hydration and solvation: The GROMOS force-field parameter sets 53A5 and 53A6. J. Comput. Chem. 2004, 25, 1656-1676.

(25) Riniker, S.; van Gunsteren, W. F. Mixing coarse-grained and fine-grained water in molecular dynamics simulations of a single system. J. Chem. Phys. 2012, 137, No. 044120.

(26) Riniker, S.; Eichenberger, A. P.; van Gunsteren, W. F. Solvating atomic level fine-grained proteins in supra-molecular level coarsegrained water for molecular dynamics simulations. Eur. Biophys. J. 2012, 41, 647-661.

(27) Orsi, M.; Sanderson, W. E.; Essex, J. W. Permeability of Small Molecules through a Lipid Bilayer: A Multiscale Simulation Study. J. Phys. Chem. B 2009, 113, 12019-12029.

(28) Michel, J.; Orsi, M.; Essex, J. W. Prediction of Partition Coefficients by Multiscale Hybrid Atomic-Level/Coarse-Grain Simulations. J. Phys. Chem. B 2008, 112, 657-660.

(29) Orsi, M.; Ding, W.; Palaiokostas, M. Direct Mixing of Atomistic Solutes and Coarse-Grained Water. J. Chem. Theory Comput. 2014, 10, 4684-4693. 
(30) Orsi, M. Comparative assessment of the ELBA coarse-grained model for water. Mol. Phys. 2014, 112, 1566-1576.

(31) Delle Site, L.; Praprotnik, M. Molecular systems with open boundaries: Theory and simulation. Phys. Rep. 2017, 693, 1-56.

(32) Zavadlav, J.; Marrink, S. J.; Praprotnik, M. Multiscale Simulation of Protein Hydration Using the SWINGER Dynamical Clustering Algorithm. J. Chem. Theory Comput. 2018, 14, 1754-1761. (33) Shadrack Jabes, B.; Klein, R.; Delle Site, L. Structural Locality and Early Stage of Aggregation of Micelles in Water: An Adaptive Resolution Molecular Dynamics Study. Adv. Theory Simul. 2018, 1, 1800025.

(34) Brodskaya, E. N.; Mudzhikova, G. V. Molecular dynamics simulation of AOT reverse micelles. Mol. Phys. 2006, 104, 36353643.

(35) Mudzhikova, G. V.; Brodskaya, E. N. Molecular simulation of an Aerosol OT reverse micelle: 1. The shape and structure of a micelle. Colloid J. 2006, 68, 729-737.

(36) Mudzhikova, G. V.; Brodskaya, E. N. Molecular simulation of an Aerosol OT reverse micelle: 2. Energy and kinetic characteristics. Colloid J. 2006, 68, 738-742.

(37) Brown, D.; Clarke, J. H. R. Molecular dynamics simulation of a model reverse micelle. J. Phys. Chem. 1988, 92, 2881-2888.

(38) Nevidimov, A. V.; Razumov, V. F. The monoatomic solvent model in molecular dynamics study of reverse micelles. High Energy Chem. 2014, 48, 202-205.

(39) Nevidimov, A. V.; Razumov, V. F. Molecular dynamics simulations of AOT reverse micelles self-assembly. Mol. Phys. 2009, 107, 2169-2180.

(40) Braun, D.; Schmollngruber, M.; Steinhauser, O. Rotational dynamics of water molecules near biological surfaces with implications for nuclear quadrupole relaxation. Phys. Chem. Chem. Phys. 2016, 18, 24620-24630.

(41) Schmollngruber, M.; Braun, D.; Oser, D.; Steinhauser, O. Dielectric depolarisation and concerted collective dynamics in AOT reverse micelles with and without ubiquitin. Phys. Chem. Chem. Phys. 2016, 18, 3606-3617.

(42) Feller, S. E.; MacKerell, A. D. An Improved Empirical Potential Energy Function for Molecular Simulations of Phospholipids. J. Phys. Chem. B 2000, 104, 7510-7515.

(43) Shinoda, W.; DeVane, R.; Klein, M. L. Multi-property fitting and parameterization of a coarse grained model for aqueous surfactants. Mol. Simul. 2007, 33, 27-36.

(44) Eichenberger, A. P.; Huang, W.; Riniker, S.; van Gunsteren, W. F. Supra-Atomic Coarse-Grained GROMOS Force Field for Aliphatic Hydrocarbons in the Liquid Phase. J. Chem. Theory Comput. 2015, 11, 2925-2937.

(45) Reith, D.; Meyer, H.; Müller-Plathe, F. Mapping Atomistic to Coarse-Grained Polymer Models Using Automatic Simplex Optimization To Fit Structural Properties. Macromolecules 2001, 34, $2335-2345$.

(46) Vierros, S.; Österberg, M.; Sammalkorpi, M. Aggregation response of triglyceride hydrolysis products in cyclohexane and triolein. Phys. Chem. Chem. Phys. 2018, 20, 27192-27204.

(47) Li, H.; Chowdhary, J.; Huang, L.; He, X.; MacKerell, A. D., Jr.; Roux, B. Drude Polarizable Force Field for Molecular Dynamics Simulations of Saturated and Unsaturated Zwitterionic Lipids. J. Chem. Theory Comput. 2017, 13, 4535-4552.

(48) Essmann, U.; Perera, L.; Berkowitz, M. L.; Darden, T.; Lee, H.; Pedersen, L. G. A smooth particle mesh Ewald method. J. Chem. Phys. 1995, 103, 8577-8593.

(49) Hess, B.; Bekker, H.; Berendsen, H. J. C.; Fraaije, J. G. E. M. LINCS: A linear constraint solver for molecular simulations. J. Comput. Chem. 1997, 18, 1463-1472.

(50) Miyamoto, S.; Kollman, P. A. Settle: An analytical version of the SHAKE and RATTLE algorithm for rigid water models. J. Comput. Chem. 1992, 13, 952-962.

(51) Bussi, G.; Donadio, D.; Parrinello, M. Canonical sampling through velocity rescaling. J. Chem. Phys. 2007, 126, No. 014101.
(52) Parrinello, M.; Rahman, A. Polymorphic transitions in single crystals: A new molecular dynamics method. J. Appl. Phys. 1981, 52, $7182-7190$

(53) Hub, J. S.; de Groot, B. L.; van der Spoel, D. g_wham-A Free Weighted Histogram Analysis Implementation Including Robust Error and Autocorrelation Estimates. J. Chem. Theory Comput. 2010, 6, 3713-3720.

(54) Kumar, S.; Rosenberg, J. M.; Bouzida, D.; Swendsen, R. H.; Kollman, P. A. THE weighted histogram analysis method for freeenergy calculations on biomolecules. I. The method. J. Comput. Chem. 1992, 13, 1011-1021.

(55) Trzesniak, D.; Kunz, A.-P.; van Gunsteren, W. F. A Comparison of Methods to Compute the Potential of Mean Force. ChemPhysChem 2006, 8, 162-169.

(56) Aucejo, A.; Burguet, M. C.; Munoz, R.; Marques, J. L. Densities, Viscosities, and Refractive Indices of Some n-Alkane Binary Liquid Systems at 298.15 K. J. Chem. Eng. Data 1995, 40, 141-147.

(57) Ortega, J.; González, C.; Galván, S. Vapor-Liquid Equilibria for Binary Systems Composed of a Propyl Ester (Ethanoate, Propanoate, Butanoate) + an n-Alkane (C7, C9). J. Chem. Eng. Data 2001, 46, 904-912.

(58) Bonhorst, C. W.; Althouse, P. M.; Triebold, H. O. Esters of Naturally Occurring Fatty Acids - Physical Properties of Methyl, Propyl, and Isopropyl Esters of C6 to C18 Saturated Fatty Acids. Ind. Eng. Chem. 1948, 40, 2379-2384.

(59) Morawetz, E. Enthalpies of vaporization of n-alkanes from C12 to C20. J. Chem. Thermodyn. 1972, 4, 139-144.

(60) Hossenlopp, I.; Scott, D. Vapor heat capacities and enthalpies of vaporization of five alkane hydrocarbons. J. Chem. Thermodyn. 1981, 13, 415-421.

(61) Baev, A. K. Specific Intermolecular Interactions of Organic Compounds; Springer Berlin Heidelberg, 2012; p 294.

(62) Lide, D. R., Ed.; Physical Constants of Organic Compounds. In CRC Handbook of Chemistry and Physics; 85th Ed.; CRC Press: Boca Raton, Florida, US, 2004.

(63) Shrestha, L. K.; Shrestha, R. G.; Oyama, K.; Matsuzawa, M.; Aramaki, K. Structural Investigation of Diglycerol Polyisostearate Reverse Micelles in Organic Solvents. J. Phys. Chem. B 2009, 113, 12669-12679.

(64) Vierros, S.; Sammalkorpi, M. Phosphatidylcholine reverse micelles on the wrong track in molecular dynamics simulations of phospholipids in an organic solvent. J. Chem. Phys. 2015, 142, No. 094902.

(65) Vierros, S.; Sammalkorpi, M. Role of hydration in phosphatidylcholine reverse micelle structure and gelation in cyclohexane: a molecular dynamics study. Phys. Chem. Chem. Phys. 2015, 17, 14951-14960.

(66) Patra, M.; Karttunen, M.; Hyvönen, M. T.; Falck, E.; Lindqvist, P.; Vattulainen, I. Molecular Dynamics Simulations of Lipid Bilayers: Major Artifacts Due to Truncating Electrostatic Interactions. Biophys. J. 2003, 84, 3636-3645.

(67) Feller, S. E.; Pastor, R. W.; Rojnuckarin, A.; Bogusz, S.; Brooks, B. R. Effect of Electrostatic Force Truncation on Interfacial and Transport Properties of Water. J. Phys. Chem. 1996, 100, 1701117020.

(68) Alper, H. E.; Bassolino, D.; Stouch, T. R. Computer simulation of a phospholipid monolayer-water system: The influence of long range forces on water structure and dynamics. J. Chem. Phys. 1993, 98, 9798-9807.

(69) Schreiber, H.; Steinhauser, O. Cutoff size does strongly influence molecular dynamics results on solvated polypeptides. Biochemistry 1992, 31, 5856-5860.

(70) de Jong, D. H.; Baoukina, S.; Ingólfsson, H. I.; Marrink, S. J. Martini straight: Boosting performance using a shorter cutoff and GPUs. Comput. Phys. Commun. 2016, 199, 1-7.

(71) Shrestha, L. K.; Dulle, M.; Glatter, O.; Aramaki, K. Structure of Polyglycerol Oleic Acid Ester Nonionic Surfactant Reverse Micelles in Decane: Growth Control by Headgroup Size. Langmuir 2010, 26, 7015-7024. 
(72) Debye, P.; Prins, W. Micellar dispersion of $\alpha$-monoglycerides in benzene and chlorobenzene. J. Colloid Sci. 1958, 13, 86-98.

(73) Debye, P.; Coll, H. The association of $\alpha$-monoglycerides in nonaqueous solvents. J. Colloid Sci. 1962, 17, 220-230.

(74) Penttilä, P.; Vierros, S.; Utriainen, K.; Carl, N.; Rautkari, L.; Sammalkorpi, M.; Österberg, M. Phospholipid-based reverse micelle structures in vegetable oil modified by water content, free fatty acid and temperature. Langmuir 2019, 35, 8373-8382.

(75) Lehtinen, O.-P.; Nugroho, R. W. N.; Lehtimaa, T.; Vierros, S.; Hiekkataipale, P.; Ruokolainen, J.; Sammalkorpi, M.; Österberg, M. Effect of temperature, water content and free fatty acid on reverse micelle formation of phospholipids in vegetable oil. Colloids Surf., B 2017, 160, 355-363.

(76) Shrestha, R. G.; Shrestha, L. K.; Ariga, K.; Abe, M. Reverse Micelle Microstructural Transformations Induced by Surfactant Molecular Structure, Concentration, and Temperature. J. Nanosci. Nanotechnol. 2011, 11, 7665-7675. 